\title{
Computational Study of Metal-Free Magnetism and Spin-Dependent Seebeck Effect in Silicene Nanoribbons with Zigzag and Klein Edges
}

\author{
Xingyi Tan $\mathbb{D}^{1,2}$ Gang Xu ${ }^{10},{ }^{1}$ Youchang Jiang, ${ }^{1}$ and Dahua Ren ${ }^{2}$ \\ ${ }^{1}$ Department of Physics, Chongqing Three Gorges University, Wanzhou 404100, China \\ ${ }^{2}$ School of Information Engineering, Hubei Minzu University, Enshi 445000, China \\ Correspondence should be addressed to Xingyi Tan; tanxy@sanxiau.edu.cn
}

Received 8 September 2021; Revised 6 January 2022; Accepted 26 January 2022; Published 12 February 2022

Academic Editor: Sefer Bora Lisesivdin

Copyright (c) 2022 Xingyi Tan et al. This is an open access article distributed under the Creative Commons Attribution License, which permits unrestricted use, distribution, and reproduction in any medium, provided the original work is properly cited.

Nanoribbons based on low-dimensional materials are potential candidates for nanoscale spintronics devices. Here, some ferromagnetic silicene nanoribbons with zigzag and Klein edges ( $N$-ZKSiNRs) are constructed. It is demonstrated that the $N$ ZKSiNRs with various widths $(N)$ are placed in various spin-resolved electronic situations. With the increase of the width parameter $N$ from 4 to 19 , the $N$-ZKSiNRs pass from the indirect-gap bipolar magnetic semiconducting state (BMS) to the bipolar spin-gapless semiconductor (BSGS) and eventually to half-metallicity (HM). Moreover, applying a temperature gradient through the nanoribbons leads to spin-dependent current with the opposite flowing and spin orientations, demonstrating the spindependent Seebeck effect (SDSE). Besides, it was found that the BSGS phase is superior to the BMS and HM for generating SDSE. These findings confirm that the ZKSiNRs are promising choices for spin caloritronics devices.

\section{Introduction}

As a combination of spintronics and thermoelectronics, spin caloritronics performs an essential task in the growth of basic sciences and new low-power consumption technologies [1-6]. Many advanced influences have been discovered in spin caloritronics in recent years, such as the spin-dependent Seebeck effect (SDSE) [7-10], thermal spin-filtering effect (TSFE) [11-13], the spin-Seebeck diode effect [14-20], and the thermal giant magnetoresistance effect (TGMRE) $[21,22]$. To date, various studies have been performed around the mentioned effects in the graphene, silicene, and black phosphorus nanoribbons with armchair or zigzag edges. For example, Zeng et al. [3] indicated that a temperature discrepancy between the drain and the source could produce opposite spin currents in magnetized zigzag graphene nanoribbons (GNRs). Yang and colleagues verified the thermal spin transport features of zigzag-edged silicene nanoribbons (ZSiNRs) and demonstrated that they could have a huge thermal magnetoresistance and a high TSFE
[23]. In our past studies, we utilized GNRs and silicene nanoribbons (SiNRs) with armchair or zigzag edges to construct spin caloritronics devices and realize marvelous thermal spin transport features such as SDSE [15, 24-26] and TSFE [24-26]. However, it was indicated that the analysis of the SDSE on zigzag SiNRs (ZSiNRs) with reconstructed edges, i.e., the Klein edge and the pentagon-heptagon, is rare. Indeed, these two kinds of reconstructed edges for zigzag GNRs have been seen during the experiments [27, 28], and their substantial impacts on the electronic structures and magnetic states of ZGNRs have been demonstrated theoretically $[29,30]$. Especially, Ding and Wang [31] found that the silicene nanoribbons with zigzag and Klein edges are more stable than unreconstructed zigzag edges under the medium and H-rich environments. Therefore, in the current work, the thermal spin-related transport characteristic and electronic structures of ZKSiNRs were investigated based on $\mathrm{ab}$ initio computations incorporated with nonequilibrium Green's function technique and demonstrated that the ZKSiNRs could have stable ferromagnetic states, and the 
SDSE could also be achieved. According to the obtained results, ZKSiNRs can be adopted to construct spin caloritronics devices.

\section{Calculation Method and Model}

Here, the device designs and theoretical methods are briefly introduced. The $\mathrm{N}$-ZKSiNRs in the present designs have one zigzag edge and one Klein edge (see Figures 1(a) and 1(b)), while two $\mathrm{H}$ atoms saturate both edges. The number of $\mathrm{Si}$ rows through the ZSiNR width is indicated by $N$, which changes from 4 to 19. As presented in Figure 1(c), two-probe spin caloritronics devices were constructed using the $\mathrm{N}$-ZKSiNRs ( say $N=4$ ). The right and left contacts are semiinfinite ZKSiNRs, while the mid-dispersion area includes five modules of ZKSiNRs. We concentrate on the spin currents driven through the temperature gradient, $\Delta T$, between the source temperature $T_{\mathrm{L}}$ and the drain one $T_{\mathrm{R}}$, i.e., $\Delta T=T_{L}-T_{R}$.

All the computations were accomplished with the Atomistix ToolKit (ATK) package [32], implementing spin density functional theory incorporated with nonequilibrium Green's function approach. The double-zeta-polarized (DZP) basis set was adopted to accomplish the geometry optimization and electronic structure computations, while the generalized gradient approximation method $[33,34]$ was utilized to obtain the exchange-correlation potential. The cutoff energy was chosen as 75 Hartree, while a Monkhorst-Pack $1 \times 1 \times 100 k$-mesh was selected. In the Landauer-Büttiker formulation, the spin-related currents of the devices were determined with the following equation [35]:

$$
I^{\uparrow(\downarrow)}=\frac{e}{h} \int_{-\infty}^{\infty}\left\{T^{\uparrow(\downarrow)}(E)\left[f_{L}\left(E, T_{L}\right)-f_{R}\left(E, T_{R}\right)\right]\right\} \mathrm{d} E,
$$

where $e$ refers to the electron charge, $h$ indicates the constant of Planck, and $T_{L(R)}$ stands for the left (right) electrode's temperature. $f_{L(R)}\left(E, T_{L(R)}\right)$ describes the left (right) electrode's mean Fermi-Dirac distribution:

$$
f_{L(R)}\left(E, T_{L(R)}\right)=\left\{1+\exp \left[E-\frac{\mu_{L(R)}}{k_{B} T_{L(R)}}\right]\right\}^{-1},
$$

where $\mu_{L(R)}$ describes the left (right) electrode's chemical potential and $k_{B}$ stands for the Boltzmann constant. $T^{\uparrow(\downarrow)}(E)$ denotes the spin-related transport coefficient, which is obtained as

$$
T^{\uparrow(\downarrow)}(E)=\operatorname{Tr}\left[\Gamma_{L} G^{r} \Gamma_{R} G^{a}\right],
$$

where $G^{r(a)}$ stands for Green's function retarded in the midarea, while $\Gamma_{L(R)}$ denotes the coupling matrix for the left (right) electrode.

\section{Results and Discussion}

First, we certify the magnetic ground state of the $\mathrm{N}$-ZKSiNRs $(N=4-19)$. To this end, we take into account the total energies of three different magnetic phases, i.e., nonmagnetic (NM), antiferromagnetic (AFM), and ferromagnetic (FM) phases. The energy difference $E_{\mathrm{FA}}=\mathrm{E}_{\mathrm{FM}}-\mathrm{E}_{\mathrm{AFM}}$ and

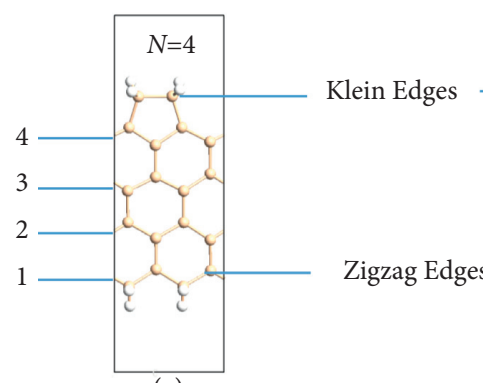

(a)

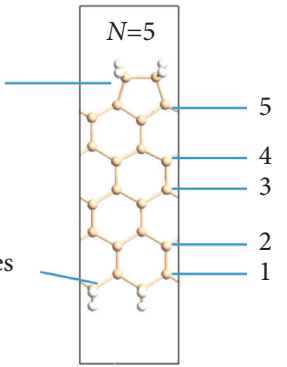

(b)

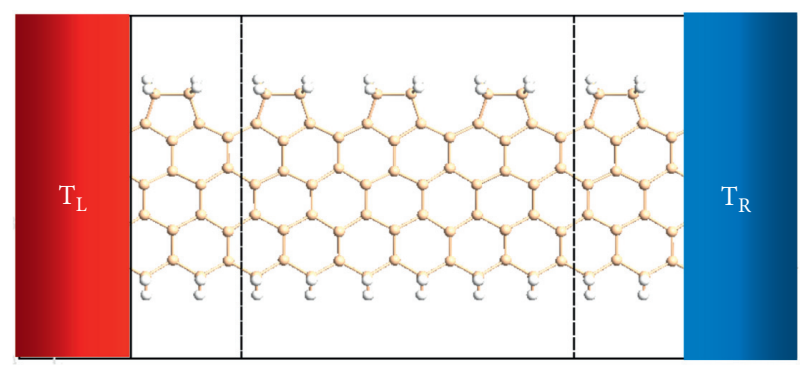

(c)

Figure 1: (a, b) Optimal structure of the $N$-ZKSiNRs (say $N=4$ and 5 ), in which silicon and hydrogen atoms are described by the gold and white balls, respectively. (c) Schematic description of the thermal spin device using $N$-AGNRs (say $N=4$ ).

$E_{\mathrm{FN}}=\mathrm{E}_{\mathrm{FM}}-\mathrm{E}_{\mathrm{NM}}$, where $\mathrm{E}_{\mathrm{NM}}, \mathrm{E}_{\mathrm{AFM}}$, and $\mathrm{E}_{\mathrm{FM}}$ expressing the total energies of NM, AFM, and FM phases between two edges of the silicene nanoribbons, are shown in Figure 2(a). It is clear that all $\mathrm{E}_{\mathrm{FA}}$ and $\mathrm{E}_{\mathrm{FN}}$ are less than zero. These calculated results demonstrated that the FM state is the ground magnetic state of the $N-Z K S i N R s(N=4-19)$. Then, the band structures of the $N$-ZKSiNRs $(N=4-19)$ were verified, as presented in Figure 3. The band structures of nanoribbons vary significantly with the increase of $N$. For $N=4-8$, the conduction band minimum (CBM) depended on the spin-down states and was located in the line of $\Gamma-Z$, above the Fermi level $\left(E_{F}\right)$, and the spin-up states below $E_{\mathrm{F}}$ are considered the valence band maximum (VBM) and located in the line of $\Gamma-Z$ at different points of the CBM. The valence and conduction bands have reverse spin polarities when they tend to $E_{\mathrm{F}}$. Besides, the spin-related bands of these ZKSiNRs have narrow gaps near $E_{\mathrm{F}}$. The mentioned features demonstrate that the above ZKSiNRs are in the indirect-gap BMS [36-40]. The ferromagnetic formation is kept unchanged while increasing the nanoribbon width, while the band structures around the Fermi level vary significantly. For $N=9-16$, both the VBM of the spin-up channel and the CBM of the spin-down channel lie at the line of $\Gamma-Z$ with different points, and the bandgaps are less than $0.1 \mathrm{eV}$. Based on the studies performed by Wang [41] and $\mathrm{Hu}$ et al. [42], the bandgap near $0.1 \mathrm{eV}$ or smaller than $0.1 \mathrm{eV}$ can be described as "gapless." Therefore, the band structure can be considered as gapless, indicating that these ZKSiNRs exhibit a BSGS status. For $N=17-19$, the spin-up bands display semiconducting features as the spin-down ones are metallic. The mentioned ZKSiNRs are normally half-metallic (HM) and can be utilized to construct high-efficiency thermal spin 


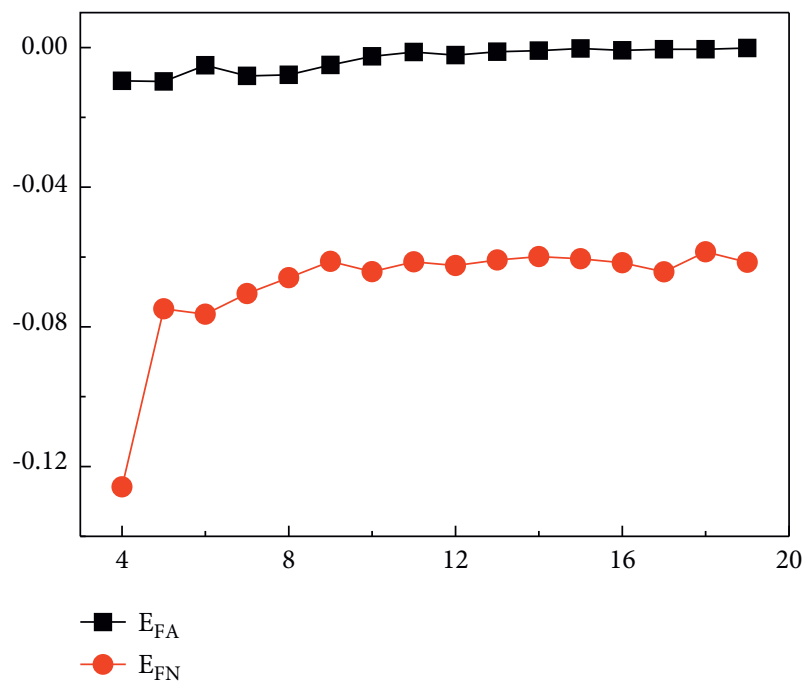

(a)

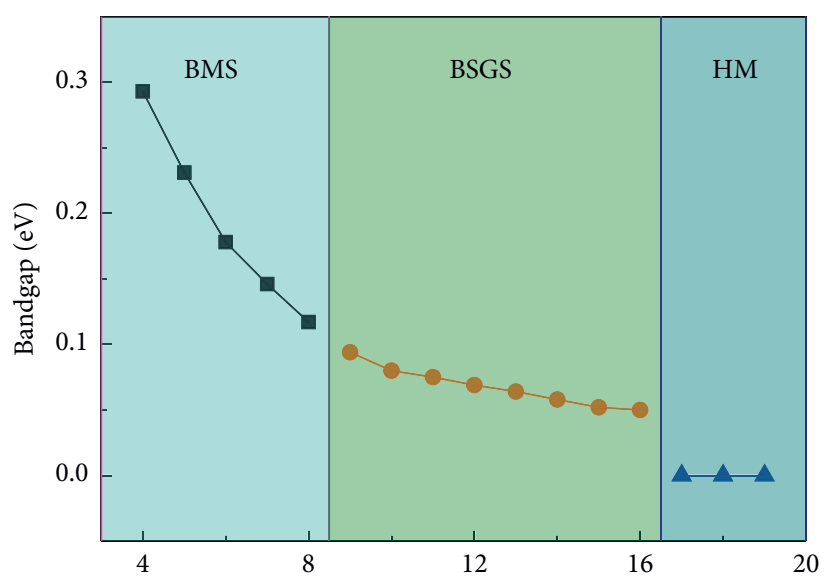

(b)

Figure 2: (a) The energy difference $\mathrm{E}_{\mathrm{FM}-\mathrm{AFM}}$ and $\mathrm{E}_{\mathrm{FM}-\mathrm{NM}}$ of the $N$-ZKSiNRs $(N=4-19)$. (b) Change in the bandgap energy in terms of the width of the $N$-ZKSiNRs $(N=4-19)$.

filters. To further confirm the HM state, we calculated and displayed the spin-dependent charge densities of the ZKSiNRs $(N=17-19)$, as shown in Figure 4. Note that the major contribution to the states on the Fermi level is due to spin-up electrons, confirming the HM state. To summarize, ZKSiNRs can have three states while increasing the width, including the indirect-gap BMS, BSGS state, and HM state, as shown in Figure 2(b).

In order to explore SDSE, thermal spin transport features of ZKSiNRs should be verified. Figure 5 presents the spinrelated currents within the $N$-ZKSiNR (taking $N=4,9,16$, and 19 as an example) devices versus $T_{L}$ and $\Delta T$. For the 4ZKSiNR device, there are no spin-up currents $\left(I_{\text {up }}\right)$ when $T_{L}<200 \mathrm{~K}$ and no spin-down currents $\left(I_{\mathrm{dn}}\right)$ when $T_{L}<250 \mathrm{~K}$ for three $\Delta T$ values, indicating that no thermal-caused spinrelated currents are produced in these ranges of $T_{L}$ for various values of the temperature difference $(\Delta T)$. This means that there exists a threshold temperature $T_{\text {th }}$ at around $200 \mathrm{~K}$ for $I_{\text {up }}$ and $250 \mathrm{~K}$ for $I_{\mathrm{dn}}$, respectively. When $T_{L}>T_{\text {th }}$, both $I_{\text {up }}$ and $I_{\text {dn }}$ grow significantly with the increase of $T_{L}$. Nevertheless, their orientations are opposite, i.e., $I_{\mathrm{up}}$ is negative, while $I_{\mathrm{dn}}$ is positive. This is certainly due to the SDSE $[14,16,22]$. Moreover, by increasing the value of $\Delta T$, the spin-related currents increase as well. The spin-related currents in terms of $\Delta T$ curves are presented in Figure 5(b), for $T_{L}=300,350$, and $400 \mathrm{~K}$. These curves demonstrate that the spin-related currents are approximatively symmetric around the zero-current axis and strong within a wide domain of temperature gradients. Accordingly, the spinrelated currents in terms of $\Delta$ T curves can confirm the SDSE. Although the devices of 9-ZKSiNRs and 16-ZKSiNRs have similar rules with 4 -ZKSiNRs, they have smaller $T_{\text {th }}$ and larger $I_{\mathrm{up}}$ and $I_{\mathrm{dn}}$, as shown in Figures 5(c)-5(f). Besides, as shown in Figures $5(\mathrm{~g})$ and $5(\mathrm{~h}), I_{\mathrm{dn}}$ of the 19-ZKSiNR device has similar rules with $4-Z K S i N R s$, while $T_{\text {th }}$ for $I_{\text {up }}$ is equal to zero.
In order to explain the physical mechanism of the above phenomena, the corresponding spin-related transmissions of $N$-ZKSiNRs are presented in Figure 6, taking $N=4,9,16$, and 19, for example. For 4-ZKSiNRs (shown in Figure 6(a)), the spin-up and spin-down transmissions are approximately distributed symmetrically in the downside and upside of the Fermi level, providing two independent spin-conducting channels. According to formulas (1) and (2), the spin-dependent currents depend on the transmission spectra and the difference of the Fermi-Dirac distribution between two electrodes. The number of electrons with energies higher than the Fermi level flows from the hotter electrode to the lower one because the electron distribution of the hotter electrode is higher than that of the lower one, causing a spindown current. For the same reason, the number of holes with energies lower than the Fermi energy flows from the hotter electrode to the lower one, too, causing the spin-up current. Therefore, applying a temperature gradient across the nanoribbons leads to opposite flow orientations for the spindown and spin-up currents, demonstrating the appearance of the SDSE [14, 16], as presented in Figures 5(a) and 5(b). For 9-ZKSiNRs and 16-ZKSiNRs (shown in Figures 6(b) and 6(c)), the spin-dependent transmissions have similar characteristics with $N=4$, except for the smaller bandgaps, resulting in smaller $T_{\mathrm{th}}$, as shown in Figures 5(c)-5(f). Therefore, the SDSE can also be obtained. For 19-ZKSiNRs (shown in Figure 6(d)), there is just a single spin channel crossing the Fermi level, indicating the related HM and thermal spin-filtering features. However, the TSFE is weak, and the SDSE occurring for the bandgap of the spin-up electron is tiny. It was found that $T_{\text {th }}$ has a positive correlation with the bandgaps, i.e., the bigger the bandgaps, the larger $T_{\mathrm{th}}$. This rule implies SDSE in larger temperature ranges and larger spin-dependent currents in BSGS materials than BMS materials. Therefore, the following conclusion can be drawn: (i) the BSGSs with zero bandgaps are the 


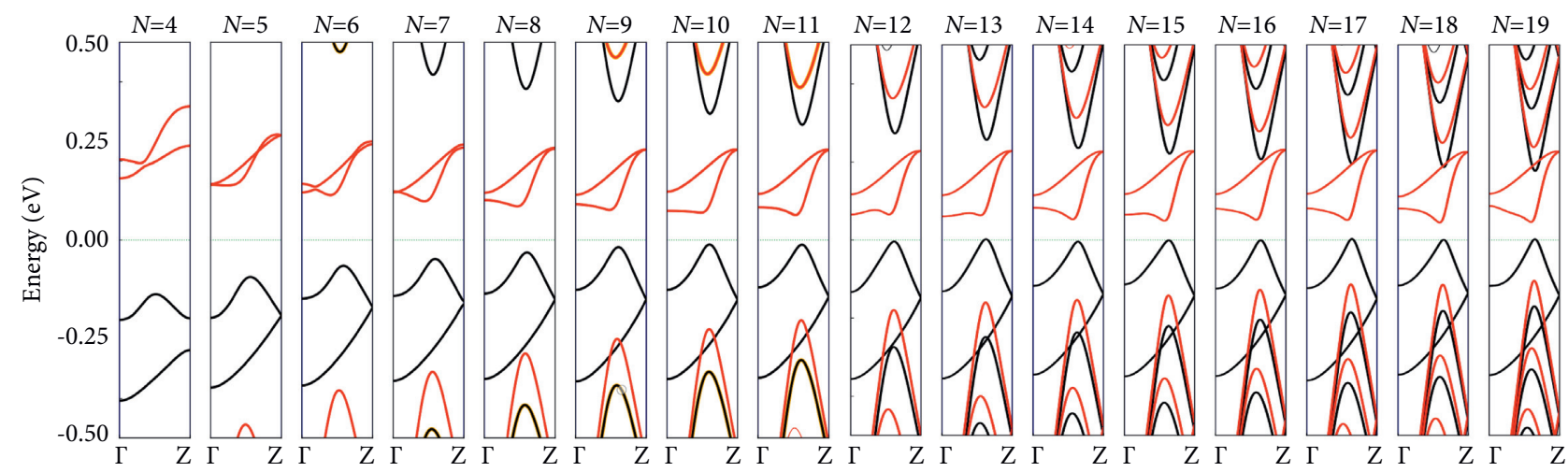

FIgURE 3: Spin-dependent band structures of the $N$-ZKSiNRs $(N=4-19)$. The black and red lines represent spin-up and spin-down electrons, respectively.

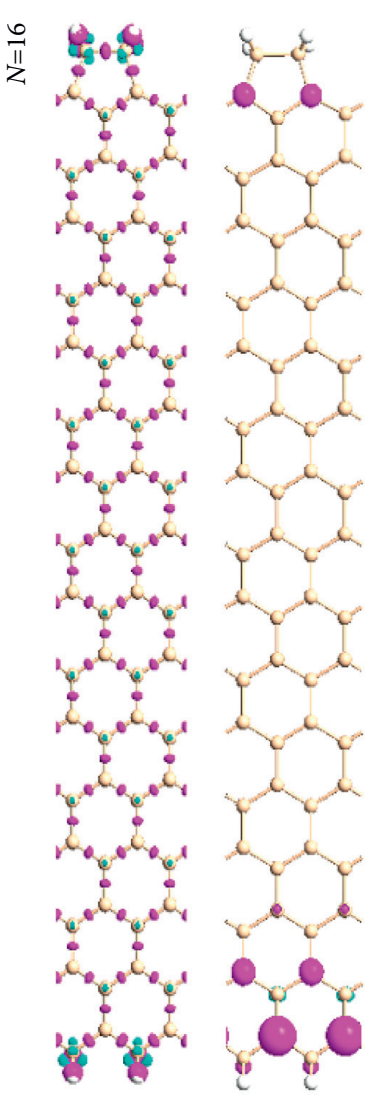

(a)

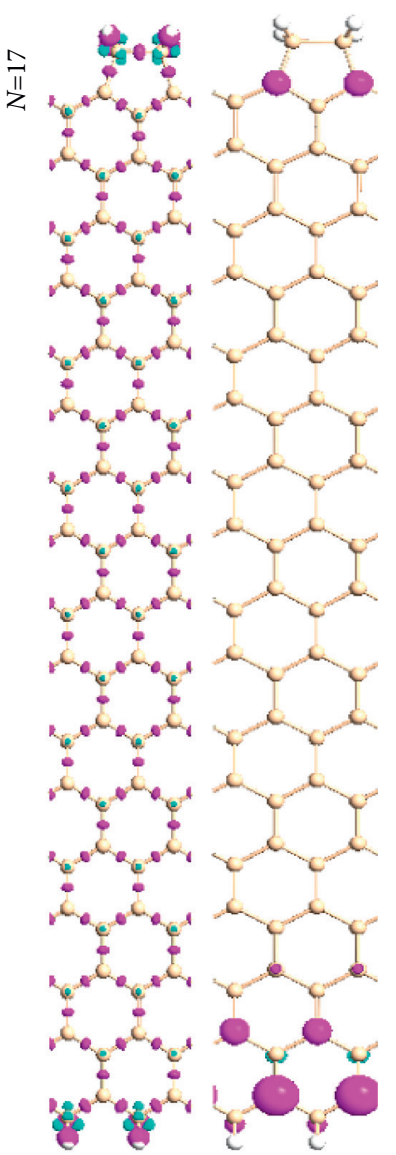

(c)

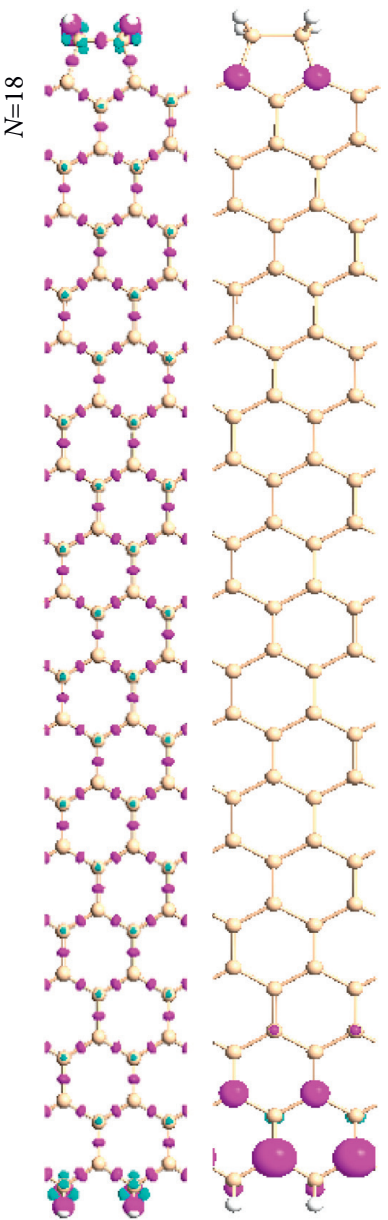

(e)

(f)

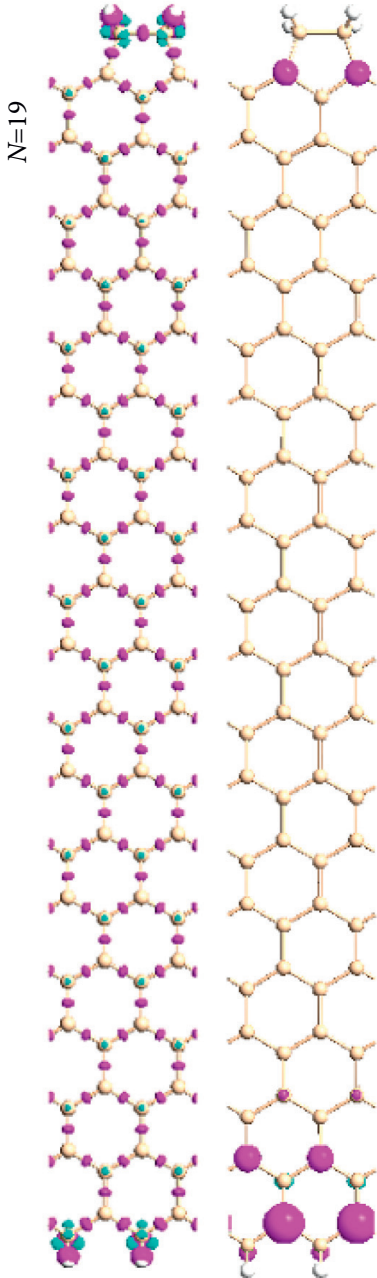

(g)

(h)

Figure 4: Spatial electronic charge distribution (up + down) (a, c, e, and g) and spatial spin distribution (up - down) (b, d, f, and h). The purple (light blue) surface represents the spin-up (spin-down) density. The value of isovalues is $0.10 \mu_{\mathrm{B}} / \AA^{3}$ in cases (a, c, e, and g). The value of isovalues is $0.02 \mu_{\mathrm{B}} / \AA^{3}$ in cases $(\mathrm{b}, \mathrm{d}, \mathrm{f}$, and $\mathrm{h}$ ). 

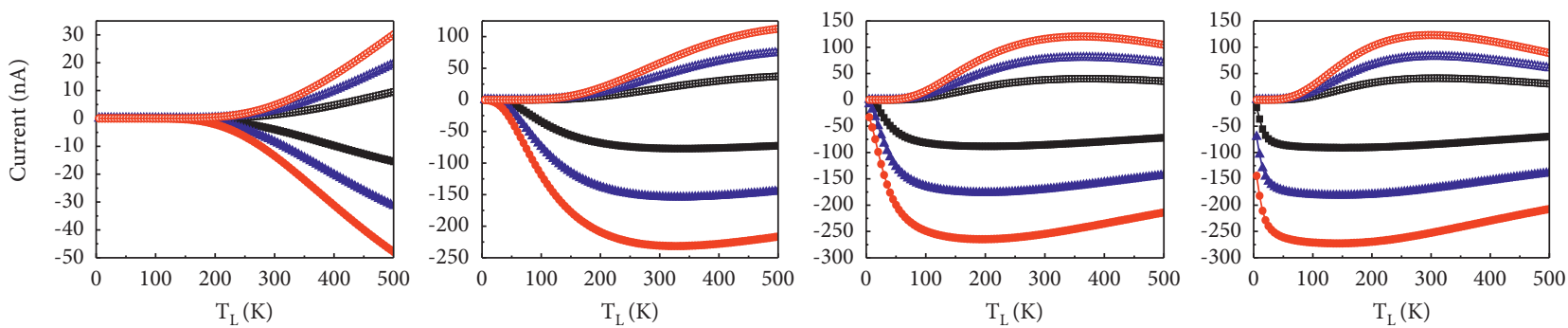

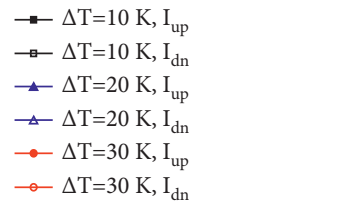

(a)

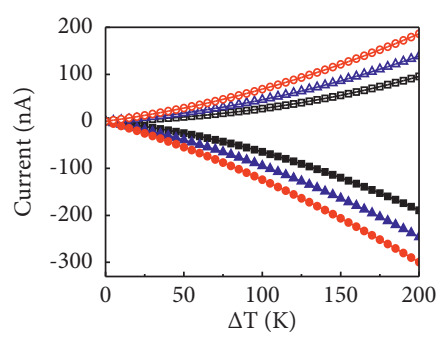

$\rightarrow \mathrm{T}_{\mathrm{L}}=300 \mathrm{~K}, \mathrm{I}_{\text {up }}$
$\longrightarrow \mathrm{T}_{\mathrm{L}}=300 \mathrm{~K}, \mathrm{I}_{\mathrm{dn}}$
$\longleftarrow \mathrm{T}_{\mathrm{L}}=350 \mathrm{~K}, \mathrm{I}_{\text {up }}$
$\longleftarrow \mathrm{T}_{\mathrm{L}}=350 \mathrm{~K}, \mathrm{I}_{\mathrm{dn}}$
$\rightarrow \mathrm{T}_{\mathrm{L}}=400 \mathrm{~K}, \mathrm{I}_{\text {up }}$
$\rightarrow \mathrm{T}_{\mathrm{L}}=400 \mathrm{~K}, \mathrm{I}_{\mathrm{dn}}$

(b)

$$
\begin{aligned}
& \rightarrow \Delta \mathrm{T}=10 \mathrm{~K}, \mathrm{I}_{\mathrm{up}} \\
& \square \Delta \mathrm{T}=10 \mathrm{~K}, \mathrm{I}_{\mathrm{dn}} \\
& \longleftarrow \Delta \mathrm{T}=20 \mathrm{~K}, \mathrm{I}_{\mathrm{up}} \\
& \longleftarrow \Delta \mathrm{T}=20 \mathrm{~K}, \mathrm{I}_{\mathrm{dn}} \\
& \rightarrow \Delta \mathrm{T}=30 \mathrm{~K}, \mathrm{I}_{\mathrm{up}} \\
& \rightarrow \Delta \mathrm{T}=30 \mathrm{~K}, \mathrm{I}_{\mathrm{dn}}
\end{aligned}
$$

(c)

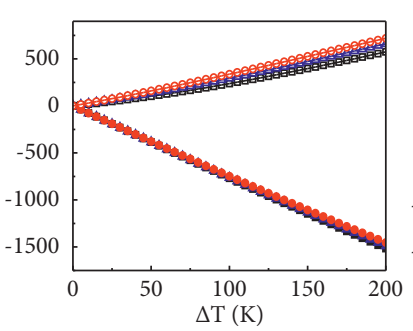

$$
\begin{aligned}
& \rightarrow \mathrm{T}_{\mathrm{L}}=300 \mathrm{~K}, \mathrm{I}_{\text {up }} \\
& \rightarrow \mathrm{T}_{\mathrm{L}}=300 \mathrm{~K}, \mathrm{I}_{\mathrm{dn}} \\
& \multimap \mathrm{T}_{\mathrm{L}}=350 \mathrm{~K}, \mathrm{I}_{\text {up }} \\
& \rightarrow \mathrm{T}_{\mathrm{L}}=350 \mathrm{~K}, \mathrm{I}_{\mathrm{dn}} \\
& \rightarrow \mathrm{T}_{\mathrm{L}}=400 \mathrm{~K}, \mathrm{I}_{\text {up }} \\
& \rightarrow \mathrm{T}_{\mathrm{L}}=400 \mathrm{~K}, \mathrm{I}_{\mathrm{dn}}
\end{aligned}
$$

(d)

$$
\begin{aligned}
& \rightarrow \Delta \mathrm{T}=10 \mathrm{~K}, \mathrm{I}_{\mathrm{up}} \\
& \square \Delta \mathrm{T}=10 \mathrm{~K}, \mathrm{I}_{\mathrm{dn}} \\
& -\Delta \mathrm{T}=20 \mathrm{~K}, \mathrm{I}_{\mathrm{up}} \\
& \triangle \Delta \mathrm{T}=20 \mathrm{~K}, \mathrm{I}_{\mathrm{dn}} \\
& \rightarrow \Delta \mathrm{T}=30 \mathrm{~K}, \mathrm{I}_{\mathrm{up}} \\
& \longrightarrow \Delta \mathrm{T}=30 \mathrm{~K}, \mathrm{I}_{\mathrm{dn}}
\end{aligned}
$$

(e)

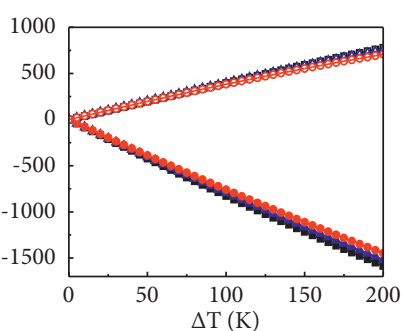

$$
\begin{aligned}
& \rightarrow \mathrm{T}_{\mathrm{L}}=300 \mathrm{~K}, \mathrm{I}_{\mathrm{up}} \\
& \rightarrow \mathrm{T}_{\mathrm{L}}=300 \mathrm{~K}, \mathrm{I}_{\mathrm{dn}} \\
& \multimap \mathrm{T}_{\mathrm{L}}=350 \mathrm{~K}, \mathrm{I}_{\mathrm{up}} \\
& \rightarrow \mathrm{T}_{\mathrm{L}}=350 \mathrm{~K}, \mathrm{I}_{\mathrm{dn}} \\
& \rightarrow \mathrm{T}_{\mathrm{L}}=400 \mathrm{~K}, \mathrm{I}_{\mathrm{up}} \\
& \rightarrow \mathrm{T}_{\mathrm{L}}=400 \mathrm{~K}, \mathrm{I}_{\mathrm{dn}}
\end{aligned}
$$

(f) $\rightarrow \Delta \mathrm{T}=10 \mathrm{~K}, \mathrm{I}_{\text {up }}$

$\rightarrow \Delta \mathrm{T}=10 \mathrm{~K}, \mathrm{I}_{\mathrm{dn}}$

$\neg \Delta \mathrm{T}=20 \mathrm{~K}, \mathrm{I}_{\text {up }}$

$\triangle \Delta \mathrm{T}=20 \mathrm{~K}, \mathrm{I}_{\mathrm{dn}}$

$\rightarrow \Delta \mathrm{T}=30 \mathrm{~K}, \mathrm{I}_{\mathrm{up}}$

$\rightarrow \Delta \mathrm{T}=30 \mathrm{~K}, \mathrm{I}_{\mathrm{dn}}$

(g)

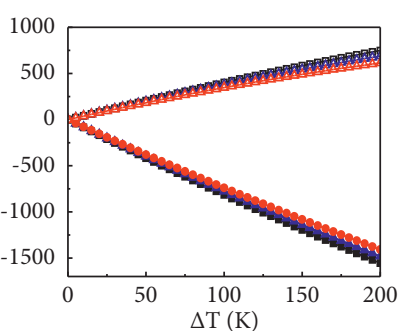

$$
\begin{aligned}
& \rightarrow \mathrm{T}_{\mathrm{L}}=300 \mathrm{~K}, \mathrm{I}_{\mathrm{up}} \\
& \rightarrow \mathrm{T}_{\mathrm{L}}=300 \mathrm{~K}, \mathrm{I}_{\mathrm{dn}} \\
& \rightarrow \mathrm{T}_{\mathrm{L}}=350 \mathrm{~K}, \mathrm{I}_{\mathrm{up}} \\
& \rightarrow \mathrm{T}_{\mathrm{L}}=350 \mathrm{~K}, \mathrm{I}_{\mathrm{dn}} \\
& \rightarrow \mathrm{T}_{\mathrm{L}}=400 \mathrm{~K}, \mathrm{I}_{\mathrm{up}} \\
& \rightarrow \mathrm{T}_{\mathrm{L}}=400 \mathrm{~K}, \mathrm{I}_{\mathrm{dn}}
\end{aligned}
$$

(h)

Figure 5: Spin-up currents $\left(I_{\text {up }}\right)$ and spin-down currents $\left(I_{\mathrm{dn}}\right)$ in terms of $T_{L}$ for the $N$-ZKSiNR devices at selected temperature gradients: (a) $N=4$, (c) $N=9$, (e) $N=16$, and (g) $N=19 . I_{\text {up }}$ and $I_{\text {dn }}$ in terms of $\Delta T$ for selected values of $T_{L}$ : (b) $N=4$, (d) $N=9$, (f) $N=16$, and (h) $N=19$.

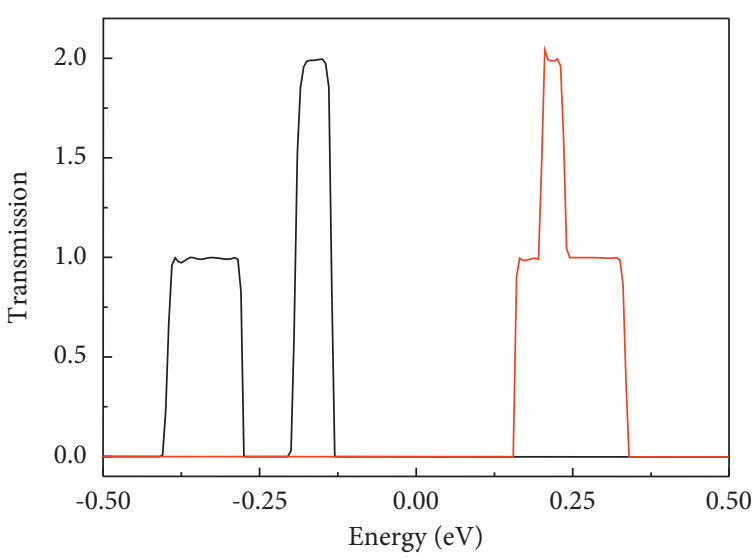

(a)

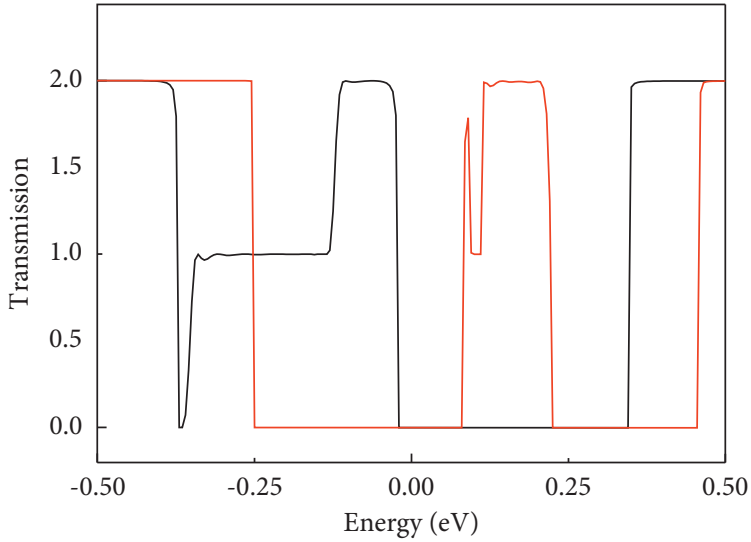

(b)

FIgure 6: Continued. 


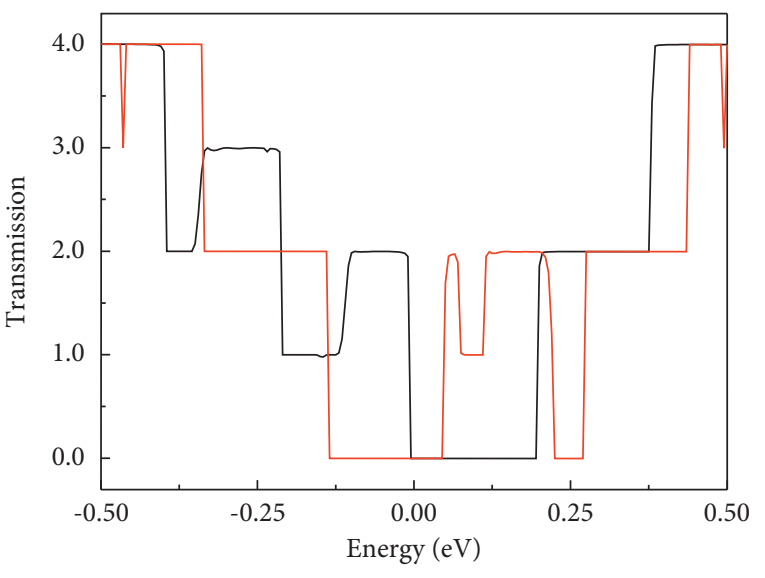

(c)

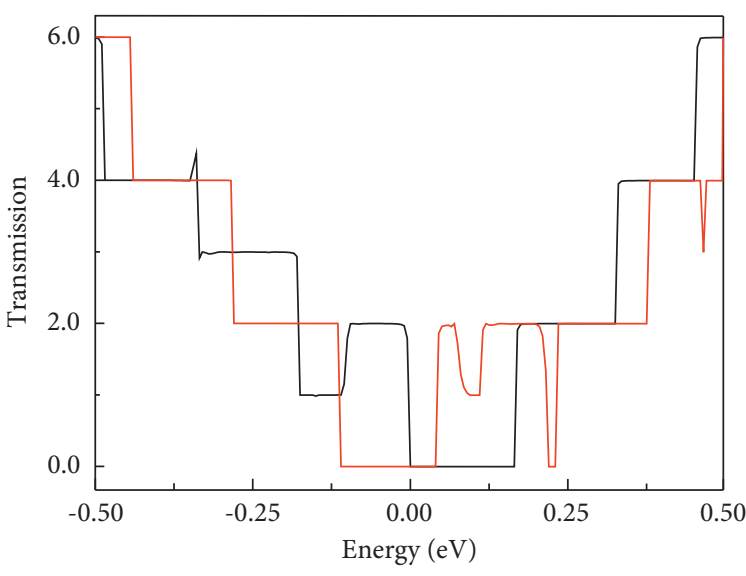

(d)

Figure 6: Spin-related transmission spectra of the $N$-ZKSiNR devices: (a) $N=4$, (b) $N=9$, (c) $N=16$, and (d) $N=19$, in which spin-down and spin-up are described by the solid black and red lines.

ideal candidate materials for generating SDSE; (ii) the HMs with one bigger bandgap are the ideal candidate materials for generating TSFE.

\section{Conclusion}

In short, the electronic structures and thermal spin-related transport features of some $\mathrm{N}$-ZKSiNRs were verified through first-principle computations incorporated with nonequilibrium Green's function. Firstly, it was demonstrated that the $\mathrm{N}$-ZKSiNRs move from an indirect-gap BMS to the BSGS state and finally to the HM one by increasing the nanoribbon width variable $N$ from 4 to 19 . Secondly, the SDSE can appear by generating a temperature gradient using the mentioned ZKSiNRs. Finally, BSGSs with zero bandgaps and the HM with one bigger bandgap are ideal candidates for generating SDSE and TSFE, respectively. Generally, the mentioned discoveries demonstrate that $N$-ZKSiNRs can be utilized to construct thermal spin nanodevices.

\section{Data Availability}

The data used to support the findings of this study are available from the corresponding author upon request.

\section{Conflicts of Interest}

The authors declare that they have no conflicts of interest.

\section{Acknowledgments}

This work was supported by the National Natural Science Foundation of China (Grant no. 11864011), in part by the Science and Technology Research Program of Chongqing Municipal Education Commission (KJQN202101204), and in part by the Educational Commission of Hubei Province of China (T201914).

\section{References}

[1] S. R. Boona, R. C. Myers, and J. P. Heremans, "Spin caloritronics," Energy \& Environmental Science, vol. 7, no. 3, pp. 885-910, 2014.

[2] G. E. W. Bauer, E. Saitoh, and B. J. van Wees, "Spin caloritronics," Nature Materials, vol. 11, no. 5, pp. 391-399, 2012.

[3] M. Zeng, Y. Feng, and G. Liang, "Graphene-based spin caloritronics," Nano Letters, vol. 11, no. 3, pp. 1369-1373, 2011.

[4] K. Uchida, J. Xiao, H. Adachi et al., "Spin Seebeck insulator," Nature Materials, vol. 9, no. 11, pp. 894-897, 2010.

[5] C. M. Jaworski, J. Yang, S. Mack, D. D. Awschalom, J. P. Heremans, and R. C. Myers, "Observation of the spinSeebeck effect in a ferromagnetic semiconductor," Nature Materials, vol. 9, no. 11, pp. 898-903, 2010.

[6] K. Uchida, S. Takahashi, K. Harii et al., "Observation of the spin Seebeck effect," Nature, vol. 455, no. 7214, pp. 778-781, 2008.

[7] A. Slachter, F. L. Bakker, J.-P. Adam, and B. J. van Wees, "Thermally driven spin injection from a ferromagnet into a non-magnetic metal," Nature Physics, vol. 6, no. 11, pp. 879-882, 2010.

[8] J.-C. Le Breton, S. Sharma, H. Saito, S. Yuasa, and R. Jansen, "Thermal spin current from a ferromagnet to silicon by Seebeck spin tunnelling," Nature, vol. 475, no. 7354, pp. 82-85, 2011.

[9] A. Torres, M. P. Lima, A. Fazzio, and A. J. R. da Silva, "Spin caloritronics in graphene with Mn," Applied Physics Letters, vol. 104, 2014.

[10] B. Z. Rameshti and A. G. Moghaddam, "Spin-dependent Seebeck effect and spin caloritronics in magnetic graphene," Physical Review B, vol. 91, 2015.

[11] G. Ding, M. Wei, G. Surucu, Z. Liang, and X. Wang, "Transition metal-doped janus monolayer SMoSe with excellent thermal spin filter and spin Seebeck effect," Applied Surface Science, vol. 491, pp. 750-756, 2019.

[12] X.-J. Gao, P. Zhao, and G. Chen, "Thermal spin-filtering, magnetoresistance effects and thermal spin logic gate in $\mathrm{Mn}$ oligoporphyrin-based molecular device," Organic Electronics, vol. 62, pp. 277-283, 2018. 
[13] H.-H. Fu and K.-L. Yao, "Perfect thermal spin filter and pure spin thermoelectric generator based on a laterally coupled double quantum-dot array," Epl, vol. 103, 2013.

[14] D.-D. Wu, G.-F. Du, and H.-H. Fu, "Spin-dependent Seebeck effect, and spin-filtering and diode effects in magnetic boronnitrogen nanotube heterojunctions," Journal of Materials Chemistry C, vol. 8, no. 13, pp. 4486-4492, 2020.

[15] X. Q. Tang, X. M. Ye, X. Y. Tan, and D. H. Ren, "Metal-free magnetism, spin-dependent Seebeck effect, and spin-Seebeck diode effect in armchair graphene nanoribbons," Scientific Reports, vol. 8, p. 927, 2018.

[16] D.-D. Wu, Q.-B. Liu, H.-H. Fu, and R. Wu, "How to realize a spin-dependent Seebeck diode effect in metallic zigzag $\gamma$-graphyne nanoribbons?" Nanoscale, vol. 9, no. 46, pp. 18334-18342, 2017.

[17] Z. Q. Zhang, Y. R. Yang, H. H. Fu, and R. Wu, "Design of spinSeebeck diode with spin semiconductors," Nanotechnology, vol. 27, Article ID 505201, 2016.

[18] H.-H. Fu, L. Gu, and D.-D. Wu, "A spin-Seebeck diode with a negative differential spin-Seebeck effect in a hydrogen-terminated zigzag silicene nanoribbon heterojunction," Physical Chemistry Chemical Physics, vol. 18, no. 18, pp. 12742-12747, 2016.

[19] H.-H. Fu, D.-D. Wu, L. Gu, M. Wu, and R. Wu, "Design for a spin-Seebeck diode based on two-dimensional materials," Physical Review B, vol. 92, 2015.

[20] S. Borlenghi, W. Wang, H. Fangohr, L. Bergqvist, and A. Delin, "Designing a spin-seebeck diode," Physical Review Letters, vol. 112, Article ID 047203, 2014.

[21] D.-D. Wu and H.-H. Fu, "Spin-Seebeck effect and thermal colossal magnetoresistance in the narrowest zigzag graphene nanoribbons," Nanotechnology, vol. 32, 2021.

[22] H. H. Fu, D. D. Wu, Z. Q. Zhang, and L. Gu, "Spin-dependent Seebeck effect, thermal colossal magnetoresistance and negative differential thermoelectric resistance in zigzag silicene nanoribbon heterojunciton," Scientific Reports, vol. 5, Article ID 10547, 2015.

[23] X. F. Yang, H. L. Wang, X. K. Hong et al., "Tunable halfmetallic properties and spin Seebeck effects in zigzag-edged graphene nanoribbons adsorbed with $\mathrm{V}$ atom or $\mathrm{V}$-benzene compound," Organic Electronics, vol. 24, pp. 80-88, 2015.

[24] X. Tan, X. Xu, L. Ding, and Y. He, "Linear and nonlinear thermal spin transport properties of zigzag alpha-graphyne nanoribbons with $\mathrm{sp}(2)-\mathrm{sp}(3)$ edges," Chemical Physics Letters, vol. 777, 2021.

[25] X. Tan, L. Ding, G.-F. Du, and H.-H. Fu, "Spin caloritronics in two-dimensional CrI3/NiCl2 van derWaals heterostructures," Physical Review B, vol. 103, 2021.

[26] J. Xiao, X. Tan, X. Ye, X. Tang, and D. Ren, “Spin-dependent Seebeck effect, spin-dependent Seebeck diode, thermal spin filtering and figure of merit of nitrophenyl diazonium functionalized graphene," Organic Electronics, vol. 63, pp. 1-6, 2018.

[27] K. Suenaga and M. Koshino, "Atom-by-atom spectroscopy at graphene edge,” Nature, vol. 468, no. 7327, pp. 1088-1090, 2010.

[28] C. O. Girit, J. C. Meyer, R. Erni et al., "Graphene at the edge: stability and dynamics," Science, vol. 323, no. 5922, pp. 1705-1708, 2009.

[29] P. Koskinen, S. Malola, and H. Häkkinen, "Self-passivating edge reconstructions of graphene," Physical Review Letters, vol. 101, Article ID 115502, 2008.

[30] P. Wagner, C. P. Ewels, J.-J. Adjizian et al., "Band gap engineering via edge-functionalization of graphene nanoribbons," Journal of Physical Chemistry C, vol. 117, no. 50, pp. 26790-26796, 2013.

[31] Y. Ding and Y. Wang, "Electronic structures of reconstructed zigzag silicene nanoribbons," Applied Physics Letters, vol. 104, 2014.

[32] M. Brandbyge, J. L. Mozos, P. Ordejon, J. Taylor, and K. Stokbro, "Density-functional method for nonequilibrium electron transport," Physical Review B, vol. 65, 2002.

[33] J. P. Perdew and Y. Wang, "Accurate and simple analytic representation of the electron-gas correlation energy," Physical Review B, vol. 45, no. 23, pp. 13244-13249, 1992.

[34] J. P. Perdew, K. Burke, and M. Ernzerhof, "Generalized gradient approximation made simple," Physical Review Letters, vol. 77, no. 18, pp. 3865-3868, 1996.

[35] J. H. Zhang, X. X. Li, and J. L. Yang, "SiN-SiC nanofilm: a nano-functional ceramic with bipolar magnetic semiconducting character," Applied Physics Letters, vol. 104, 2014.

[36] X. Li, X. Wu, Z. Li, J. Yang, and J. G. Hou, "Bipolar magnetic semiconductors: a new class of spintronics materials," Nanoscale, vol. 4, no. 18, pp. 5680-5685, 2012.

[37] V. V. Bannikov and A. L. Ivanovskii, "Ab initio search for novel bipolar magnetic semiconductors: layered $\mathrm{YZnAsO}$ doped with $\mathrm{Fe}$ and Mn," JETP Letters, vol. 96, no. 11, pp. 735-738, 2013.

[38] L. Yuan, Z. Li, and J. Yang, "Hydrogenated bilayer wurtzite SiC nanofilms: a two-dimensional bipolar magnetic semiconductor material," Physical Chemistry Chemical Physics, vol. 15, no. 2, pp. 497-503, 2013.

[39] X. Li and J. Yang, "First-principles design of spintronics materials," National Science Review, vol. 3, no. 3, pp. 365-381, 2016.

[40] X. L. Wang, "Proposal for a new class of materials: spin gapless semiconductors," Physical Review Letters, vol. 100, Article ID 156404, 2008.

[41] X. Hu, W. Zhang, L. Sun, and A. V. Krasheninnikov, "Goldembedded zigzag graphene nanoribbons as spin gapless semiconductors," Physical Review B, vol. 86, 2012.

[42] Y. Imry and R. Landauer, "Conductance viewed as transmission," Reviews of Modern Physics, vol. 71, no. 2, pp. S306-S312, 1999. 